\title{
Wiki Justice, Social Ergonomics, and Ethical Collaborations
}

\author{
By Jonah Bossewitch, John Frankfurt, Alexander Sherman, with \\ Robin D.G. Kelley
}

Introduction

"We don't stop with asking what a tool does. We ask about what kind of people we become when we use it."1

The capacity for technology to promote certain modes of behavior has long been a topic of interest for social and cultural scholars. ${ }^{2}$ Software in particular plays an obvious role in influencing creativity and production, as studies on topics ranging from word processing to power point have demonstrated. ${ }^{3}$ Theorists claim that technology and the media it brokers are "transforming the way we know and think," impacting our cognitive styles much like language itself does. ${ }^{4}$

In the Information Age, more and more of our inter-personal communications are negotiated through the intermediaries of software. The structure and form of the interactions suggested by these environments are important in understanding their effect on society at large, and especially within an educational setting. Many of the 
communication challenges encountered which faculty and students encounter in the classroom resemble the communication challenges that are encountered within organizations, between organizations and their constituents, between companies and their customers, or a government and its citizens.

In this essay we explore various theoretical, pedagogical, and historical aspects of wikis focusing on three questions as points of departure-_"What is a wiki?"; "How do you teach with a wiki?" and finally "What is the point of a wiki?"

Our chapter begins by exploring the question "What is a wiki?" Here, we propose a model which locates wikis within the university's pedagogy-technology context and describes their social and other impact. Our model postulates three layers: One, the variety of pedagogical and technological environments a university chooses to support; Two, the sets of rules, policies, and content workflows that distinguish a social software (wikis versus blogs, forums, tagging, etc.); Three, the social, cognitive, emotional, and personal impacts the engagement fosters. This model thus offers a powerful way to define and understand wikis.

Our second question, "How do you teach with a wiki?" introduces a case study, a particular classroom implementation of a wiki, to illustrate the model. In Spring 2005, Columbia University's Center for New Media Teaching and Learning (CCNMTL) collaborated with Professor Robin Kelley to launch a wiki in his undergraduate course "Black Movements in the U.S." Throughout the semester, eighty students iteratively developed the content of a collaborative web site about key social justice movements in the United States. Addressing the curricular challenges posed by using a wiki, we discuss 
why Kelley and CCNMTL selected the wiki platform, the advanced preparations that were necessary, and strategies for monitoring and evaluating the student work in the wiki.

With the model, the case study, and other examples of collaborative composition, we explore the historical context and significance of the wiki as a medium for writing in our third question, "What is the point of a wiki?" Specifically, how do the collaborative composition experiences of Kelley's students compare with notable collaborations from history? We explore the example of Diderot's grand eighteenth-century communal effort, Encyclopédie, or Oxford's nineteenth-century thousand-contributor dictionary project.

Has the wiki superseded these earlier techniques - can the process of constructing a social justice wiki really promote equality? Will the wiki earn an enduring place in the classroom, or will it go the way of blotting paper and fountain pens?

In an epilogue to this essay, Kelley reflects on the use of the wiki in his classroom. Additionally, he offers a personal word, comparing wikis to his expectations and prior collaborative curricular assignments, and how he plans to incorporate this type of technology into his future research and teaching. 


\section{The Model: What is a Wiki?}

\section{Essence of Engagement}

Our understanding of wikis can be enriched by looking at them in the various pedagogical and technological landscapes/contexts in which they operate. Generally speaking, new concepts are understood in relation to the network of concepts which surround them. ${ }^{5}$ In keeping with this, any examination of technologies in an educational setting also needs to take into account the curricular goals and pedagogical strategies guiding the classroom experience. Wikis belong to a family of technologies informally labeled social software. Members of this family include familiar applications such as blogs, forums, and social tagging. A deeper understanding of wikis and their distinctive features emerges from studying its relationships to similar technologies.

For example, blog and wiki software can be used to support all sorts of activities which are not commonly associated with the activities of "blogging" or "wikiing." This includes activities like sharing syllabi, publishing announcements, and distributing files. These newer tools can also provide spaces for discussions, similar to "traditional" mailing lists and discussion boards. When maintained over time, these systems effectively describe a student portfolio system. ${ }^{6}$ Some of the typical activities that these systems support range from the bureaucratic to discussion oriented, from collaboration to portfolios. $^{7}$ 
The differences between these variations and approaches derive from the types of engagement they are trying to foster. Technology should be used to support existing educational objectives and can also serve to promote certain styles of behavior and engagement. Thus while many educational objectives and activities can be supported by a variety of technical devices, the selection of a particular configuration may provide structure and direction, and encourage subtly different kinds of interaction. It is therefore useful to identify and describe environments that look superficially similar but are functionally different, as well as ones that look different but are functionally equivalent. By so doing, we will be better equipped to distinguish between raw software functionality and the varieties of engagements they support.

Culture of Use: Code $=$ Law?

Social software environments encourage particular usages, but a complete understanding of the dynamics within these communities requires an examination of the written and unwritten policies which may be stipulated, but are often not enforced by the system. Very rigid software systems constrain the degrees of freedom which users can exercise when communicating within these systems. For example, the software governing modern news publications strictly distinguishes between the roles of journalists, editors, and publishers by assigning particular capabilities to each. More flexible social software systems might combine user abilities, and the behaviors that take shape within these systems are best described as a social contract, ethical framework, or governance structure which delineates the interactions within the community. 
Wikis are an especially poignant example of how policies affect usages, since their flexibility is both their greatest strength and weakness. Mark Phillipson has developed a taxonomy of wiki usages, all of which can be supported using most wiki software. ${ }^{8}$ The purpose which the software serves — the essence of the engagement — is determined by the way its participants agree to use it. Thus, in Phillipson's "illuminated wiki," the wiki software does not prevent any user from altering the poem everyone is commenting on, but the wiki community using this tool prescribes leaving it intact, and their culture explains and enforces this. So, the software rules allow editing but the social policies do not.

In most wiki environments, there are mechanisms which allow for policy to be corrected after the fact, rather than prevented from occurring in the first place. In particular, the history and rollback feature, common in many wiki environments, changes the necessity for strictly enforced behavioral guidelines — in this respect, a degree of trust is extended to all wiki participants, although it is often tempered with the knowledge that all edits are preserved on the participant's permanent record. Only when we consider the rules embodied in the software, as well how those rules are configured and combined with the software's culture of use, can we begin to appreciate the full dynamics of these tools.

\section{Platonic Wikis}

So far we have considered wikis as a part of the family of technologies informally labeled "social software." From a technical vantage point, it is also useful to consider 
wikis in relation to their software predecessor, the Content Management System (CMS). A CMS is a set of processes and technologies designed to allow users with little technical knowledge the ability to organize, review, and publish digital content. In this respect, a wiki is also a kind of CMS where the rules are set so that anyone can edit it — anything you can see you can change.

All forms of Social Software can be described by the rules, policies, and workflows which are applied to their content. In this context we are using the term "content" in its most generic sense. From this perspective, articles, posts, comments, and replies, are all just pieces of content. What differentiates these various types of content are the different rules and policies that are applied to them, and the workflows they follow in their progression through the system. Discussion boards support the exchange of ideas between single authors, and often do not permit the revision of a post. Wikis, on the other hand, support the exchange of ideas with multiple authors, potentially edited and revised over time. Rules such as these enforce who is allowed to perform operations such as creating, editing, and publishing.

Content Management Systems permit their users to control and refine the rules which the software enforces, and are continually expanding the types of rules subject to adjustment. Such systems provide content administrators and developers the ability to create tools which enforce particular combinations of these rules according to the requirements of the situation. In a perfect CMS, which has yet to be implemented, the rules would be arbitrarily configurable, leading to the prospect of system designers who 
can focus their efforts on the deliberate arrangement and orchestration of the rules governing these environments.

To illustrate how imprecise the term "wiki" can be, consider "simple wikis"— those without categories or histories. "Simple wikis" don't group posts or ideas, and users cannot see what changes have been made or who has made them. Unlike the most common wikis today, it is hard to follow the thread of a discussion. Whatever is on the screen is the last word. Another illustration is the "despot wiki"—where the community is closed, you need to log in to participate, and then can edit only your own section. These "despot wikis" foster controlling behavior by the editor-limiting users, limiting posts, limiting change. Are all of these wikis?

We are composing this paper in Mediawiki, the same environment used by Robin Kelley's "Black Movements in the U.S.," our case-study class. One of the most commonly used wiki engines, Mediawiki powers Wikipedia. It can be configured with to offer complete open access or require users to $\log$ in, with file upload enabled or not. It also includes a discussion space for each post and automatically creates a home page for every member. The malleability of wiki software makes it very hard to pinpoint and describe across installations. Simply referring to a software package's name is often not enough to specify exactly which software rules or social policies determined the online collaboration.

With this apparatus in mind, it is easier to understand and differentiate the proliferation of systems that have emerged around these themes. Thinking in terms of rules, policies, and workflows applied to content it is possible to define the Platonic 
forms of social software: e.g. A Platonic Wiki can be defined as an environment where everyone can see anything that has been published, can edit anything they can see, and can easily create a new page. Similarly, a Platonic Blog can be defined as an environment where the author can create a new post, anyone can comment on an existing post, and posts are displayed in reverse-chronological order.

Currently, very few technologies aspire to implement the Platonic forms of any of these tools. In fact, it is the variations and riffs on these forms that are potentially the most interesting. It is pedantic to be so preoccupied with semantics that a particular piece of software can no longer be classified as a "wiki" if it supports fine-grained permissioning over different areas within the site. At the same time, identifying the ideal typical forms of these tools makes it possible to imagine the variations in rules that might inflect different behaviors amongst the participants. Figure 1.1 envisions the interplay between these distinct, yet related, social software systems. 


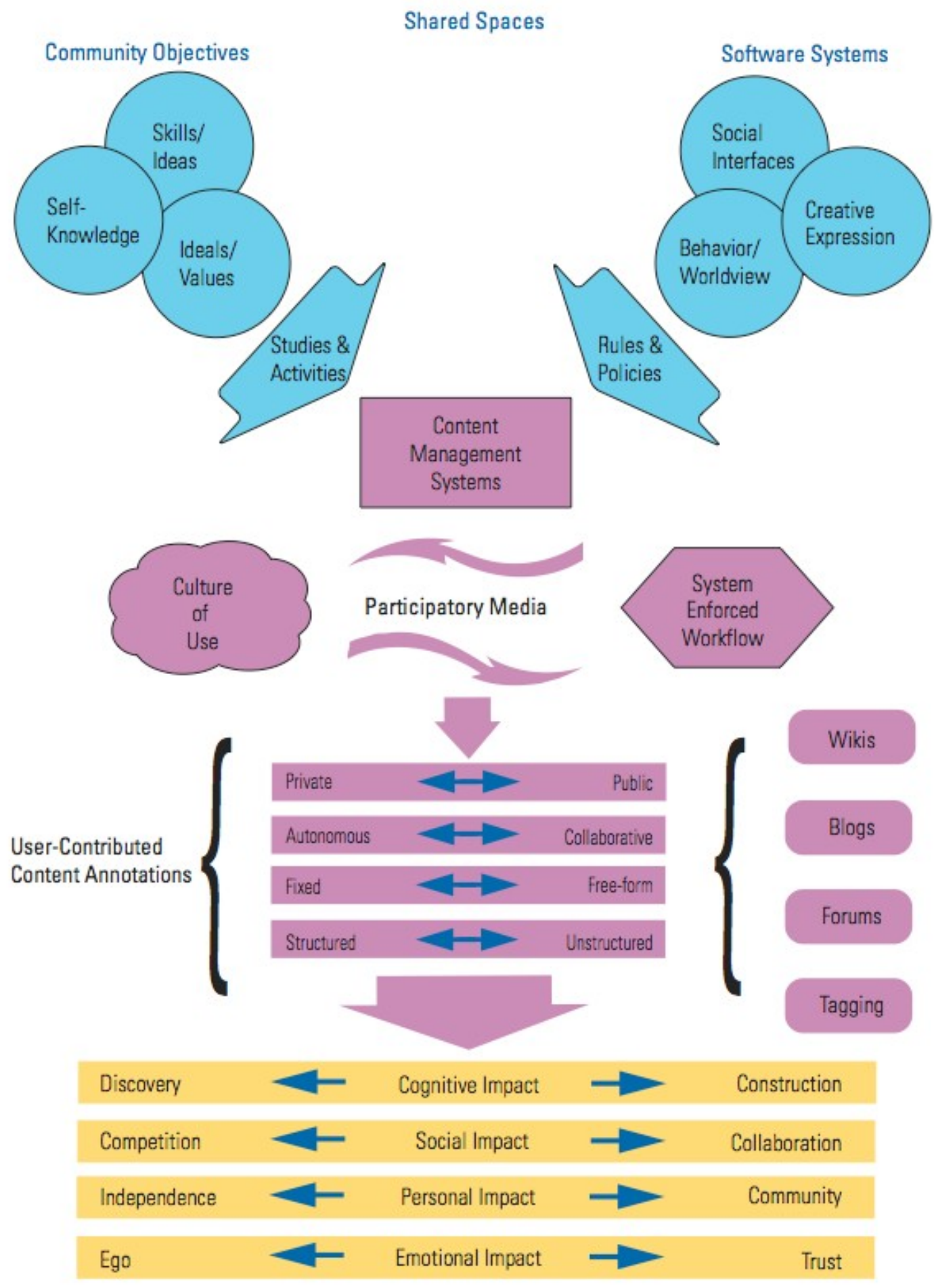

Fig. 1.1: Social Software Values 
The social software value-space postulates a continuum of values that software environments can directly affect by encouraging, facilitating, and catalyzing effects of specific types of engagements. The deliberate selection of specific policies to govern the environment will favor different types of interactions and experiences for the users within that environment. The axes of this value-space are meant to convey that these environments are capable of imparting more than subject matter. They have the potential to influence the values of the users in ways that ought to be considered by the designers of these environments.

These variations can even be seen across deployments of the very same piece of software, and are even more pronounced as we begin to vary the design of the system. Consider the differences in dynamics between two classroom-blogging situations: Contrast a situation where each individual student has her own blog, versus having the entire class share ownership and authorship of a common blog. Each of these deployments would likely be situated differently within the value-space defined above. Should we expect different degrees of autonomy, trust, and competition across these different setups?

This is not to suggest a deterministic outcome based upon the selection of a particular technological configuration. Designers of these environments should be encouraged to deliberately consider the desired outcomes, i.e., where are the participants ideally situated within this value-space, and select the technology and its corresponding configuration accordingly. At best the environment will stack the odds in favor of certain kinds of interactions; it will, not guarantee them. The obvious analogy here is to 
architects who design physical spaces with the aim of encouraging mingling or enabling mobility and flow. There is no guarantee that the final project will realize their intentions but, in fact, they often do.

\section{Social Interfaces: Software as Ideology}

Software environments now influence psychology and culture in ways that have been historically attributed to architectural works. A contributing factor to the significance of architecture is the investment of large amounts of capital. The outcome of many building projects is determined before their design occurs — they will be built, one way or another. Similarly, the construction of software environments is often driven by requirements independent of the ethical design considerations examined in this essay. As we write this, the environments that mediate communications and learning are being constructed. These systems are now responsible for mediating the communication between individuals, organizations, and institutions. The rules of engagement are becoming set in stone, or more accurately, etched in silicon. We ought to be conscious and deliberate about their form.

The term social interface captures the idea that software environments create conditions for users which shape the nature of their interactions with each other. ${ }^{9}$ Ergonomics is the study of designs intended to minimize the stress and discomfort of usage. Good hardware designs minimize physical stress, good user interfaces minimize cognitive stress, and good social interfaces minimize social stress. Examples of applications which present social interfaces include simple communications tools like 
email and news readers, social networking applications like friendster, del.icio.us, and flickr, and social software applications such as forums, blogs, and wikis.

Neither user interfaces nor social interfaces are specific to the digital age. Donald Norman describes the usability of door knobs and teapots in the language of user interfaces ${ }^{10}$, and theoretical architecture and anthropology have long described physical forms, spaces, and rituals in ways that could be described as social interfaces. The prevalence and malleability of software affords new media environments a degree of uniqueness, but this uniqueness is one of quality, not kind. ${ }^{11}$

As a corollary, since writing software is a form of creative expression it follows that the individual and community values invested in the creation of a system are almost inevitably embodied in the features which ultimately describe that system. A simple illustration of this idea is the default ability to assign a Creative Commons license using the GNU General Public License Mediawiki software, which would be a surprising default in an application produced in a proprietary setting, e.g., an Adobe product. Software is now a cultural form, expressing an ideology (in this case, the importance of the freedom of knowledge), and capturing the logic of its birthplace.

It is not surprising that wikis gestated and were born within free and open source communities. The ecology describing a software environment's creation is an important inflection point when considering the values that environment might support. This does not mean that these systems will persuade their users to adopt these values but, given our arguments above, they may induce modes of behavior which will in turn lead to a heightened awareness and adjustment of perspective. 
The case study we will now turn to is the story of the expansion and transformation of the participant's worldview. Technology was not the only influence on this educational journey, but it was the vehicle which helped them directly experience the living reality of the issues they were confronting.

The Case Study: How Do You Teach with a Wiki?

In Spring 2005, CCNMTL launched a wiki in Professor Robin Kelley's undergraduate course "Black Movements in the U.S." Kelley's class examines both historical and contemporary black activist movements for freedom, justice, equality, autonomy and self-determination. The class explores, among other things, how movements were formed and sustained; the social and historical contexts for their emergence and demise; and the impact they might have had on power, on participants in the movement, on the community at large, and on a people's vision of a liberated future. Kelley wants his class not only to study activism as a written history, but as something that is relevant and alive today. It is out of this curricular goal - to teach activism as alive and meaningful today — that the need for a wiki for this class emerged.

Throughout the semester, Kelley required all eighty students in the course, divided into groups of three to four, to iteratively develop the content of a collaborative web site about key social justice movements in New York City. In each case, students explored the broader political vision(s) of each of these movements (what are they trying to accomplish), the context for their emergence, their strategies and tactics, the impact they have had on the communities they serve as well as on struggles for social justice as a 
whole, and the kind of support they need to sustain the work they are doing. Students were required to interview organizers and conduct library research on the history and current activities on the organizations for which they were responsible. The idea to use a wiki was based on the Kelley's need to have his students work collaboratively. Additionally, because this was effectively a semester-long project, Kelley needed to be able to check in and provide feedback to the students as they were working on their projects.

The Social Justice Movements wiki, created for this class project, was a collaborative work space for the student teams to develop their organization pages. ${ }^{12}$ In order to develop and implement a wiki, Kelley approached Columbia University's Center for New Media Teaching and Learning (CCNMTL), a university resource for faculty interested in using technology in the classroom to advance specific curricular goals. ${ }^{13}$ Working with Kelley, CCNMTL initialized a wiki in development and production followed by a specific design skin for his class wiki. The next step was to add the initial content Kelley needed before introducing the wiki to the class including, among other things, instructions for the class project and an alphabetical listing of the activist organizations to be assigned to the student groups.

Following the initial work conducted by Kelley and CCNMTL, an orientation of the Social Justice wiki was given to the entire class, with the first assignment acting as training session. Students were asked to visit a robust wiki such as Wikipedia and spend some time navigating the site. Following this, the students were asked to create their user page in the class wiki. The only requirements were that their user page take advantage of some of the basic wiki functionality: embedding an image, using various text fonts, and 
creating links to both external web-sites and new pages within the wiki. After the one week that was needed for orientation and the training assignment, the students began their work on their organization pages.

As result of the painless technical demands to build a wiki page, the burden on the students for this project could be content-driven. Student team members therefore had the opportunity to contribute directly and equally to their assigned organization pages. The Social Justice wiki at this point was a password protected site, available to the class only. The "class-only" status of the wiki was meaningful as Kelley was able to encourage the class to use the wiki as a drafting space for their projects and not simply wait to publish their page at the very end of the semester. The process of researching and constructing the organization pages was useful to both the student groups to collaboratively work out the ideas, information and aesthetic of their pages together and for Kelley to provide feedback. Similarly, with the history function in the wiki that allows a user to see what changes have been made to a page a by whom, Kelley was also able to make sure that the student groups were in fact working collaboratively. In summary then, there were four elements of the wiki that were especially beneficial for Kelley's assignment:

1. The ability to introduce a new technology into the course with minimal technical training;

2. The ability for students to work collaboratively;

3. The ability for Kelley to provide feedback throughout the semester;

4. The ability for Kelley to monitor the student projects and ensure they are being constructed collaboratively. 
Towards the end of the semester, the class met to present the latest versions of their assigned organization pages. Each organization page contained information related to mission, history, membership and current activities. For instance, the page built for BlackOut Arts Collective, a grassroots coalition of artists and educators working to improve minority communities through the arts, is shown in figure 1.2.

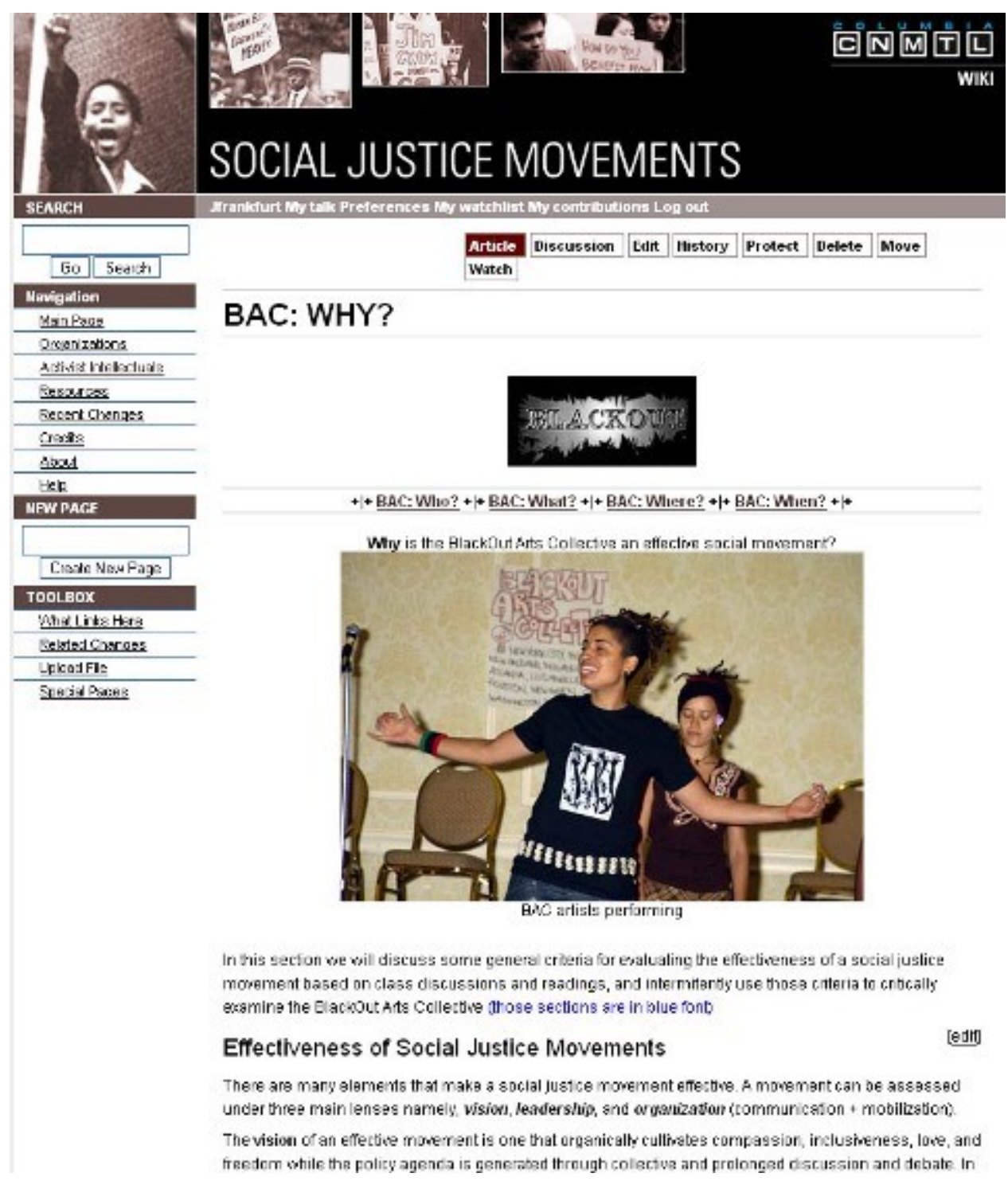

Fig. 1.2: BlackOut Arts Collective 
Here we see some other examples of organization pages in figure 1.3.
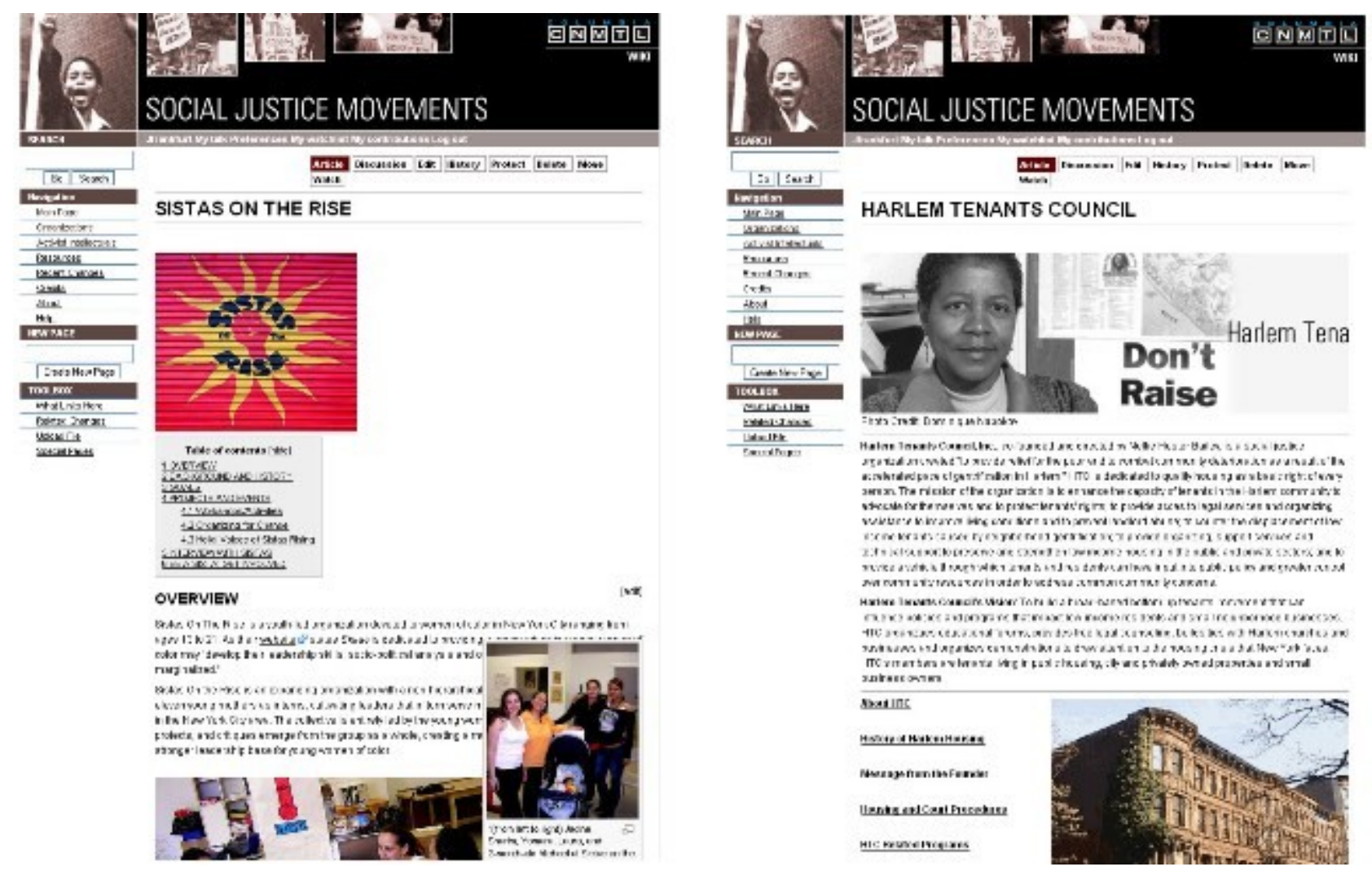

Fig. 1.3: Sistas on the Rise and Harlem Tenants Council

In addition to researching the various organizations, Kelley required the student to propose what kind of activism the groups were primarily focused on-for instance, arts, economics, or sexual identity. Following this, the class as a whole had to propose these labels, associate them with each respective group, and then use them as meta-categories 
to organize the assigned activist organizations. It is important to reiterate that while Kelley selected the organizations at the start of the semester, he provided no labels. In fact, the only means by which he sorted the organizations was alphabetically, a generic taxonomy so that all assigned groups could be located by the students while not capturing any real sense of the specific activism conducted by each group. Indeed, the kind of activism these groups are conducting is part of the take-away for the students. And because the categories had to apply not only to one group but several, all eighty members of the class had to come to an agreement on how to classify the various organizations. The labeling of all the organizations into categories was a critical moment in the collective understanding of the class that each of their pages were part of one single site.

Generally speaking, wikis are well suited for collaborative projects where the intended outcome is a cohesive whole, as opposed to a collection of independent or loosely related ideas, shown below in figures 1.4 and 1.5. Wikis are also a good tool for iteratively developing ideas over time, allowing for collaborators to revise and reorganize their contributions as themes emerge. Blogging software or a discussion board would not have allowed Kelley's class to perform these activities which were essential to the project as a whole. 


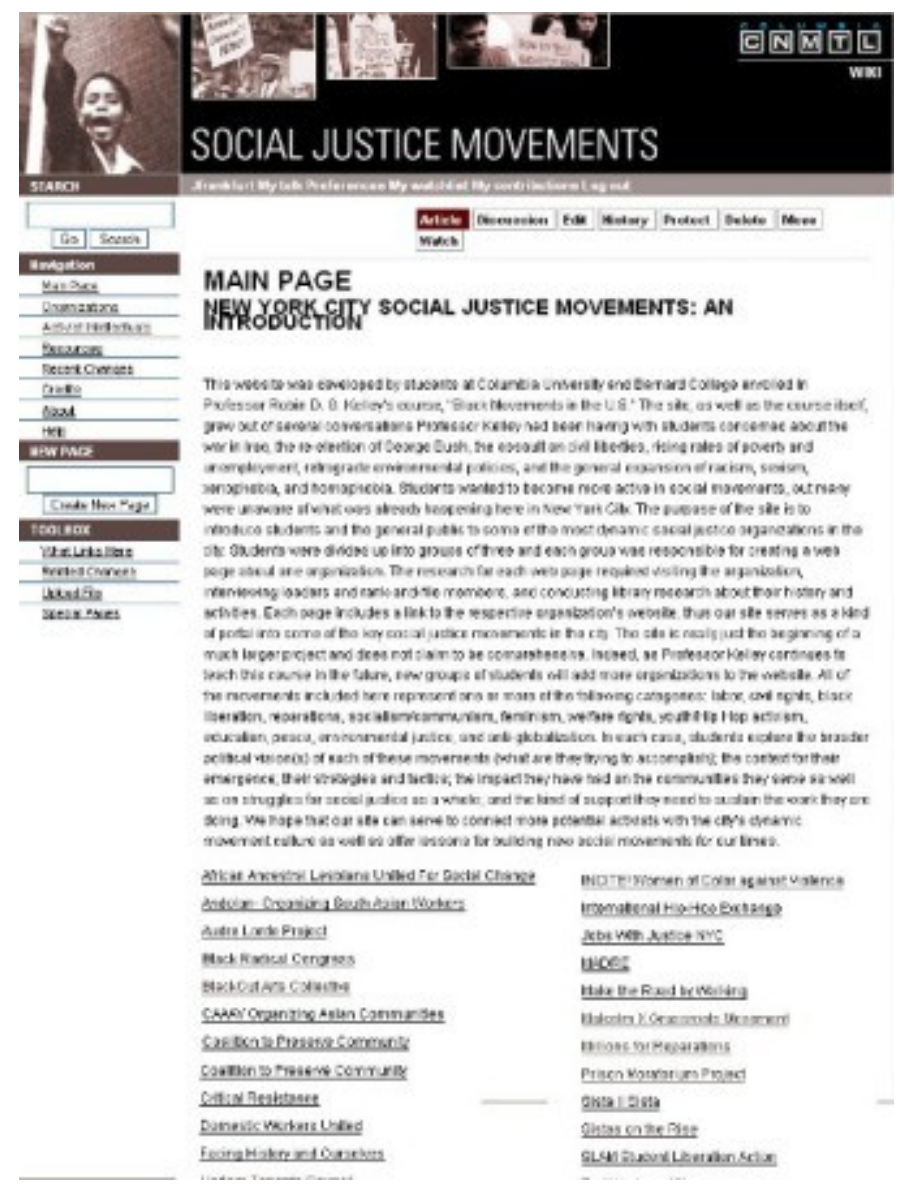

Fig. 1.4: Social Justice Main Page, February 2005, before student categories. 


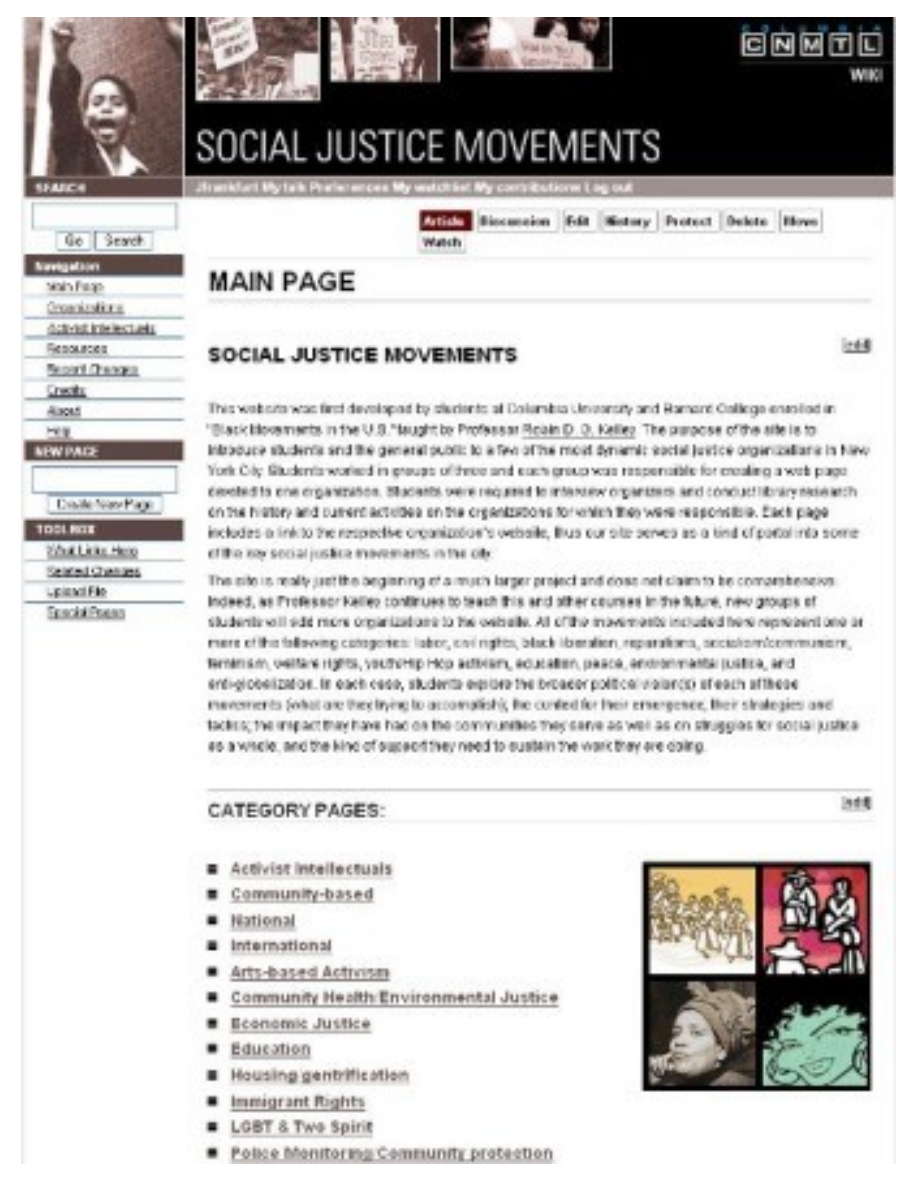

Fig. 1.5: Social Justice Main Page, February 2006, after the student categories.

Along with the design, training and implementation, Kelley and CCNMTL also developed methods to evaluate and eventually grade the wiki project. They developed four criteria for grading: the final product; response to feedback; collaboration and finally the aesthetic of the page. The most important criteria for grading was the quality of the content: that is, Kelley evaluated each organization page like one does for the traditional print paper. Following the content, the next criteria for grading was the extent to which groups responded to Kelley's feedback during the work on the project. While Kelley did 
look at each organization page like a final paper, he took advantage of the "Discussion" field — sometimes known as "Talk"—in the wiki to provide students feedback throughout the work on their project. Feedback on the organization pages ranged from the basic such as "no contact information" to the more complicated "need to better contextualize a mission statement.” Kelley's perception of how much, or how little, students took advantage of his feedback was factored into the final grade.

Collaboration was the next quality factored in grading the wiki. Grading collaboration in the wiki in some ways presents the same problems as grading class participation — especially in a large class of eighty students like "Black Movements in the U.S." Kelley and CCNMTL considered quantifying collaboration via the "History" section in the wiki where every change is logged or by introducing third party visualization tools. Finally, however, it was decided to give a grade to the project as a whole as opposed to each individual student. At the same time, the in-class presentations —where every member of the student group presents his or her organization—did gave a snap-shot of the level of collaboration in the wiki.

The final quality Kelley considered when grading was the aesthetic of the site. Given that this was an online project, Kelley encouraged the students to take advantage of the powers of this environment when building their organization pages- for instance, posting pictures of organization members or events, adding maps to show where the organization is located as well as providing links to external relevant web-sites. Similarly, the architecture of the site — where one clicks to find information — was also considered when grading. The students in Black Movements in the U.S. were not being trained to be 
webmasters, but Kelley wanted online pages that had a creative and informed navigation as opposed to simply being a long text document. In summary, Kelley evaluated and eventually graded the wiki project on the following criteria, in order of importance:

1. The content of each organization page;

2. To what extent groups responded to his feedback during the work on the project;

3. The level of collaboration in each group;

4. The aesthetic of the site as it helps one to learn about each organization.

With the work completed on the organization pages and the categories agreed upon at the end of the spring 2005 semester, the Social Justice wiki was released as a public site which anyone on the Web could view. With each organization page including a link to the respective organization's website, the Social Justice wiki now serves as a portal into some of the key social justice movements in New York City. The organization pages in the Social Justice wiki represent for some groups their first web presence of any kind.

Following the public release of the Social Justice wiki, Kelley has continued to introduce the wiki in other classes_-including the two seminars on Black Intellectuals seminars that he taught in Fall 2005 at Columbia and Harvard. Instead of focusing on organizations, as was the case in the Black Movements class taught in Spring 2005, the Columbia and Harvard seminars focused on individuals. Kelley required both seminars to work collaboratively in the Social Justice wiki space: specifically, to build pages on activist individuals and connect them to the already created categories applied for the organization pages. 
The collaborative work Kelley has his students conduct on either activist organizations or activist individuals supports one the guiding aims of his courses: that is, to present activism not only as a series of past events but as living history. For the duration of a semester, Kelley asks his students to undertake research and also gain practical experience by engaging with the contemporary world of activism. With the Social Justice wiki continuing to grow as a resource on activism, which offers potential strategies for social change, this particular wiki fosters an alternative online culture leaning towards the oppositional. The public sphere of this wiki, in this context, is defined by its distance from existing social and cultural norms that requires an active commitment and awareness of all its contributors thereby resulting in the potential to learn new means to express critical public opinion. The Social Justice wiki seeks to be a space which fosters a mode of self-creation through membership in a media-defined venue.

At the end of this essay, Kelley will discuss all the implementations as well as future plans for the Social Justice wiki. As more implementations and uses of the Social Justice wiki are planned, it becomes important to consider more not only the process entailed/generated/encouraged by the wiki but its products as well. What value, for instance, did the Spring 2005 version of the Social Justice wiki have for the Fall 2005 seminars? Another issue with the Fall 2005 implementation and its future uses raised concerned the role of the Social Justice wiki as a public workspace: is it a public web-site where all site pages can be accessed by anyone online? And should all site elements be available at all times to all members of the class? Does the growing public visibility of the Social Justice wiki - globally and in the classroom — change how students add to and 
modify the site? Do the students read more critically, contemplate more deeply, and respond and offer their own ideas more constructively? These issues and questions are ultimately at the heart of a much larger discussion about how transformative wikis and other genres of "social software" can be in both educational and popular/public contexts. Having offered a model of and discussed a case study for using a wiki, the next section considers the wiki in the context of other collaborative enterprises such as encyclopedias and dictionaries in order to explore wikis as spaces of process and product.

\section{The Context and Significance: What Is the Point of a Wiki?}

Large-scale collaborations provide rich comparisons to wikis. Encyclopedias and dictionaries often require large-scale collaboration, and there are numerous historical examples of these, even from thousands of years ago. Two more recent efforts, Encyclopédie and the Oxford English Dictionary, are both famous and well documented, and we will discuss both.

Each of these collaborative efforts reflect three key elements of Kelley's class's experience with wikis. One, an authority—teacher or editor — set the scope and wrote the rules and policies of contribution, but did not set the categories for organizing the information. Second, these efforts live (at least partially) outside the ivory tower. Kelley asked his students to research, experience, and become part of the contemporary world of activism - a directive echoing these two earlier efforts. Third, since the readers and writers belong so closely to the same community, it is difficult to distinguish author from 
audience. Bear these three elements in mind as we consider the historical precedents of Encyclopédie and the Oxford English Dictionary.

In 1745, a Parisian publisher retained two foreigners to translate an English encyclopedia into French. When it came to pre-selling copies, though, it turned out they had not completed much work. The publisher tried a new translator, but still had no success. In 1747 he engaged a French duo to work for about three years. ${ }^{14}$ Denis Diderot and Jean d'Alembert, the two new editors, sat down to look over the work and planned a significant change, namely the Encyclopédie. Rather than translating an English work, they planned to collect new information from throughout France. ${ }^{15}$ Just as Kelley did setting out with "Black Movements," identifying the research topics, the editors sketched the entire content out thematically, the two of them, and then recruited writers to fill in the sections (for example, theology or arts and crafts) with alphabetical articles to be chosen by the contributor. In this way, the entire effort was centrally planned at the beginning. In fact, each article was distinguished either "O" for contributor or "*" for editor—an early form of "log in."16

As with Kelley's class and most wikis, the readers and the writers of the Encyclopédie largely overlapped. In terms of raw numbers, when the first volumes came out in the early 1750 s, the subscribers (a large number for the time) were hardly more numerous than the contributors and staff. In fact, it was so large a collaboration that roughly $1 \%$ of Paris was contributing to the project. ${ }^{17}$ The authors and the audience (those who subscribed) both belonged to the literate and thinking folk of France, the salon set. ${ }^{18}$ Even the censorious contributed -15 of the $100+$ contributors also worked as 
government censors ${ }^{19}$ In all, more $100+$ writers contributed 72,998 articles over 26 years to create the Encyclopédie.

The Encyclopédie set the tone for later collaborative works-fostering community as much as writing a document. In this case, the community was much more formal, perhaps because eighteenth-century French society itself was formal. After the volumes starting coming out, the contributors met regularly—calling themselves Encyclopedists and meeting at Baron d'Holbach's every Thursday and Sunday. Similarly, but unusual for a wiki effort, Kelley's class also met regularly in person. (Writers about Wikipedia regularly comment on the vibrancy of the online community.) Strong social interaction supports social activism. In Kelley's class's case, activism included volunteering at social justice organizations. For the Encyclopedists, activism included atheism, erotica, and other activities deemed subversive by the French authorities of the day. In terms of activism, what better mark of service is there than serving jail time? The Encyclopédie nevertheless hit hard times when Diderot himself did "hard time" (albeit for the innocuous sounding "Letter on the Blind").

The Oxford English Dictionary serves as another landmark in the history of collaborative writing projects. It took some 70 years to publish the whole first edition. The $O E D$ is the desert island book par excellence —or rather 12 desert island books, since it was published as 12 tomes totaling 15,499 pages. Most dictionaries include guides to definition and pronunciation. In addition, the $O E D$ offers 1,827,306 quotes to illustrate every meaning of 414,825 words. ("Salt," for example, covers 14 columns over 6 pages 
—not counting "salt cote," "salt fat," or "salt like"—beginning with the pre-Norman the Conqueror: "Wip blæce, wyl eolonan on buteran, meng wyp sote, sealt, teoro." $)^{20}$

When the Unregistered Words Committee of the London Philological Society launched an effort to write the $O E D$ in 1857 , they had a rough idea that this would be big, so they adopted a new methodology that the Grimm brothers were using-recruiting volunteers to read and find different meanings. ${ }^{21}$ In practice, the $O E D$ you read has been gathered from each of these volunteers - a system employing many authors, just like a wiki. Their complex interactions were governed by slowly evolving rules, just as wikis and other CMS's have specific rules. In the case of the $O E D$, volunteers submitted their quotes of example usage of words, which were sorted by two people. (Originally, they thought 54 5-inch pigeon holes would hold all the words in English — they were off by two orders of magnitude). Then "re-sub-editors" gathered these submissions by word and by part of speech. Then sub-editors for "S" or "Q," say, gathered these chronologically and began distinguishing definitions. The editor at the top at last composed each definition and submitted it for publication.

Strict policies guided each collaborator's submissions, though the stringency of enforcement varied among editors. The first editor called these policies by their Latin name, Canones Lexicographici, setting out exactly how each volunteer should read, what centuries they should cover, even particular authors that were in short supply. ${ }^{22}$ Like wiki policies, the Rules guided how the information should be structured, and even how the foolscap paper should be formatted! 
By involving the eventual readers of the dictionary in its very writing, the Unregistered Words Committee intended a more collaborative undertaking. ${ }^{23}$ Thus they launched an Appeal to the entire English world to contribute. Two-thousand Appeals were distributed and reprinted in newspapers around the world, entitled "An Appeal to the English-Speaking and English-Reading Public to Read Books and Make Extracts."24 In this sense, the authors and audience were one and the same. ${ }^{25}$

This broad approach was so successful, the project ballooned beyond all expectations. The contract signed with Oxford University Press in 1878 (21 years into the project) stipulated 10 years more of work. It took 54 more years. The contract stated 7,000 pages. The result was 16,000 . They expected it to cost $£ 9,000$. It cost $£ 300,000$. $^{26}$ The collaboration spawns its own sort of energy, making it difficult for anyone to get his or her arms around it at the beginning. As with many collaborations, the $O E D$ team faced the question: when is enough enough?

In order to highlight the benefits of wiki technology in general, it is probably useful to contrast the historical precedents we have described with a wiki larger than that created by Kelley's class. Therefore, let's consider the familiar case of Wikipedia.

Table 1.1 Encyclopédie,OED, and Wikipedia Comparison

\begin{tabular}{|l|l|l|l|l|}
\hline & \multicolumn{1}{|c|}{ Contributors } & \multicolumn{1}{|c|}{ Entries } & Editor Effort & Years to publish \\
\hline Encyclopédie & $100+$ & 72,998 & 21 & $6 / 26$ \\
\hline OED & $\sim 1,000$ & 414,000 & $\sim 1,000$ & $\mathrm{X} / 71$ \\
\hline Wikipedia & $15,000+{ }^{27}$ & $960,000+$ & $\sim 502$ & $1 /$ ongoing \\
& & & & \\
\hline
\end{tabular}


The three collaborations resemble each other in size, namely, the number of collaborators and articles. These are each massive undertakings, engaging more people than the average person knows. ${ }^{28}$ And the expanse covered in each case exceeds any one person's polymathy. The key point to understand is that the wiki-based effort is not larger than its predecessors just because it is a wiki. In fact, as Table 1.1 demonstrates, wikis have not yet engendered collaborative writing on a different scale than preceding technology. What they do is provide a new answer to an old problem, just as the ballpoint pen answered the ink-to-paper question differently than the fountain pen did. And even though ballpoints are a lot less effort, fountain fetishists persist with their Parkers and Penguins. Looking to the future, we expect new collaboration technologies to be even less effort than wikis, but a few pockets of people — for affectation or other reasons - will likely persist with wikis.

In general, it is difficult to deny that wikis are easier to use than earlier collaborative technologies: the wiki technology automates much of the effort that went in to the historical oeuvres. From editor, to staff, to the years of compilation, the Wikipedia takes less effort. Most technologies today share this efficiency relative to their Enlightenment or Industrial analogues. The ease of use and low price spread wikis quickly, but as the qualitative and quantitative comparison to antecedents suggest, vis a vis collaborative tools, wikis are not doing anything radically new.

In other ways, wikis depart radically from previous efforts, in particular, in the opportunity for ongoing revision. Consider the "Dewey Defeats Truman" blunder. Today, that mistaken report of the election results of 1948 could be corrected instantly. This 
speed not only helps accuracy, it also encourages engagement. Unlike other collaborations, a wiki makes it possible to hit "save page" and you see the effect of your effort right away. Speed encourages engagement—quality of process—and sometimes quality of the product too. Wiki editors are instant stakeholders. You see your activism.

This historical perspective echoes our earlier theoretical perspective, that wikis are but a type of CMS — one specific family of rules and policies for organizing information. Neither theory nor history distinguishes wikis from other content systems or collaborative approaches. Our case study nevertheless does bode well for collaborative endeavors in general, however their content rules and policies are defined. The benefits of such endeavors are precisely those which became apparent in Kelley's class, namely, the role of originator, the real-world community, and the collapsing of author and audience. This last aspect of the wiki has two interesting facets.

The first facet is that of accuracy through exhaustion: these collaborative efforts are collaborative precisely because they require a massive amount of human effort. Their accuracy is judged by how exhaustively they cover the relevant bases. For example, the $O E D$ sought to plumb the depths of English, with the more citations per word the better. ${ }^{29}$

The second facet made plain by the collapse of author and audience is that of the general audience: perhaps wiki epistemology works best when the audience is general. For Diderot, his readers were his writers. The $O E D$, believe it or not, was also intended for the general public, not the philological few. And Wikipedia is the first reference for generalists, not PhDs. 
Viewed in historical context, and in light of these notable similarities, wikis no longer appear to be an aberration in the history of composition. They are not revolutionary. The advantages of collaborative writing preceded wikis and will endure long after them as well. Wikis are a great technology but they by no means offer a unique approach to composition. Consider for a minute the little magnetic words many people have on their refrigerators. They approximate a Surrealist game from 1920s — taking words out of context to find new meanings in them. The Surrealists played games like Exquisite Corpse, where one person writes down a definite or indefinite article and an adjective, the next person a noun, the third person a verb, and so on, each without looking at what the previous person wrote. The final sentence often has unexpected meaning. "Surrealist texts obtained simultaneously by several people writing from such to such a time in the same room, collaborative efforts ... brought out into the open a strange possibility of thought, which is that of its pooling"..$^{30}$ The new collaborative meaning is precisely predicated on NOT reading what the others write before editing it. In this way, you discard your personal will and meaning and succumb to a group intention. ${ }^{31}$

Which brings us back to the question, can the process of constructing a social justice wiki really promote equality? As we saw, wikis, like other collaborative efforts, value the process as much as the product - the community engendered is a major benefit of writing in them. It is not the wiki technology per se that engenders equality, but the collaborative effort on social justice. (Which raises the question: would a wiki on social injustice would promote inequity?). When we share an endeavor, perforce we share goals. Sharing goals and working together, we come to share values. The shared values and 
aspirations describe a world which we, as a group, believe to be better. Thus, wiki or no, together we make the world better.

What is a wiki? A content management system anyone can read or edit.

How do you teach a wiki? Set a topic and grade students on their ability to agree on meaningful categories.

What is the point of wiki? Instant stakeholders and a collaboration where you see the impact of your effort.

\section{Conclusion}

The internet which most people are currently familiar with is like an infinite glass wall. On one side of the wall, are a small number of people with markers, writing on the glass for the rest of the world to read. Wikis fulfill one of the original intentions of the web - bringing everyone to the same side of the glass and giving them all markers. The importance of providing individuals with this kind of autonomy and agency is exemplified in the historical discourse around the Adventure Playgrounds. ${ }^{32}$

In the detritus of the Second World War, the children of Europe played. Adapting the idea from the Danish junk playgrounds, the English let their kids loose on the sites destroyed by the Blitz. ${ }^{33}$ The children played "with building materials, discarded objects and tools, and ... build the playground according to their own ideas and for their own pleasure".${ }^{34}$ With few rules, they enjoy building a fort one day and take just as much 
pleasure in destroying it the next. Proponents of this freeform play proposed that the war had alienated children by wresting away control of their lives, and this lack of control was causing juvenile delinquency. Adventure Playgrounds offered freeform play, where the children were in charge. This exercise in control would engender broader civic participation and agency through their young lives.

If it is permissible to compare small things to large, similarly today, the commercial wars have usurped control of the internet. As browsers, we tread a battlefield of commercials. Flashing colors pop up willy nilly on our screens. We are jerked from site to site. We don't even control our own names; anonymous corporations hoard our personal information. In the late ' 90 s and early ' 00 s, it seemed any time you entered the web, you checked your personal control at the login. We were powerless, we were alienated, and we were delinquent. Is it any wonder the virus epidemic broke out so? Geeky delinquents asserted themselves, if at all, not as hollow beings, but as lost, violent souls - wreaking damage on the rest of us.

Adventure Playgrounds offered children the chance to reclaim the space around them. Wikis offer us the chance to reclaim the cyberspace around us. Once again, as silicon citizens, we determine what is written on our screens. Wikis offer the sense of control that the commercial wars blitzed. The best measure of wiki will be- not how many articles are posted, or how many edits are made, or accuracy but—civic and cyber engagement. Collaborative projects by their nature win over those who choose to engage. If wikis successfully engage people on civic issues like social justice, we may expect those folks at least to promote social justice (while still disagreeing about what it means). 
So the test of wikis will be "Is it yet easy enough to engage? Have we found the right way to work together to improve the world?"

\section{Epilogue}

By Robin D.G. Kelley

In past undergraduate courses, I always required students to collaborate on projects. Usually these collaborations took the form of classroom presentations of collective research, or collections of primary documents relevant to the class that students organize, edit, and introduce in the form of a collaborative essay. But for "Black Movements in the U.S.," I decided to try something new: to turn what would have been classroom presentations into a permanent website focused on a movement for social justice. Initially, I envisioned these sites in HTML language and went to CCNMTL to show them how to build it. It was at that initial meeting with John Frankfurt and Jonah Bossewitch that I was introduced to the wiki.

Of the eighty-plus students in my course, very few were computer savvy. Indeed, many of the students considered themselves activists and were very hesitant when I announced that they would be building websites. Only three or four students in the entire class were familiar with HTML language and had had some experience creating websites, and fewer than ten had even heard of wiki. Nevertheless, I learned some of the basics and introduced the basic syntax to the students. In addition, both John and Jonah visited the class and gave a brief but thorough presentation on the wiki. The students' first assignment was to create a personal page on the wiki site - a short autobiography along 
with photos and internal and/or external links that might be relevant. This assignment allowed students to become comfortable with the syntax and very soon they were up to speed in terms of loading images, text, and creating links to their own site or between sites under construction.

The wiki turned out to be the best teaching tool I've ever used. Students not only conducted substantial library research but the visual and audio requirements of the site compelled them to search for multimedia sources. They also had to write entries and essays about their subject matter for a public audience rather than for a professor or a teaching assistant. Thus they could not take anything for granted and had to create prose that filled in all the gaps in knowledge. More importantly, they had to create more internal and external links to names/concepts/historical events with which few general readers would be familiar. Providing links to definitions, descriptions, and contextual information was much better than simply listing a source or a footnote.

Finally, the collaborative nature of the project compelled students to make links to other groups. For example, at least three groups were working on movements attempting to dismantle the prison system. It soon became clear that certain terms were used commonly by all organizations involved, most notably, "prison industrial complex." Rather than create three different definitions of the PIC, students from three different groups decided to write one definition to which all three groups might be linked.

I was especially pleased with the way in which these projects affected the activist community at large. In some cases, the organizations for which students created wiki pages had no websites. The wiki sites became their portal to the world. The members of 
these various social justice organizations became very interested in using the sites and they, too, began to learn the wiki syntax. They wanted to use the wiki as an active site where they could add announcements for forthcoming events and possibly create space for discussion. Activists were especially drawn to the user-friendly nature of the wiki because they did not want to become dependent on a web master or web designer to create a site they could not change or alter on their own.

Next year I will be teaching at the University of Southern California (USC) and my colleagues and some of the students I have met at USC are already talking about the Social Justice web site. I'm hoping to continue building the project, first by focusing on local Los Angeles activist organizations and taking advantage of students' knowledge of the city. I plan to have students add on to the existing site. One possible outcome is that USC students might be inspired to work with the Columbia and Harvard students who have already contributed to the site not to mention the possibilities of collaboration across various social justice movements. 
${ }^{1}$ Howard Rheingold, "Rheingold's Rants.” July 4, 1998. http://www.rheingold.com/rants/ (Accessed June 13, 2005).

${ }^{2}$ Marshall McLuhan, Understanding Media: The Extension of Man. (New York: Signet Books, 1964).

${ }^{3}$ Edward R. Tufte, The Cognitive Style of Power Point: Pitching Out Corrupts Within. 2nd ed. (Cheshire, CT: Graphics Press, 2006).

${ }^{4}$ Michael H. Heim, "Heidegger and McLuhan and The Essence of Virtual Reality," in Philosophy of Technology: The Technological Condition: An Anthology, ed. Robert C. Scharff and Val Dusek (Malden, MA: Blackwell Publishers, 2003), 539-555.

5 "So in what direction will one discover the path that leads to the statesman? For we must discover it, and after having separated it from the rest we must impress one character on it; and having sampled a single different form on the other turnings we must make our mind think of all kinds of knowledge as being two forms." Plato, Statesman, ed. and trans. C.J. Rowe, (Warminster, England: Aris \& Phillips, 1995), 258c.

${ }^{6}$ A number of colleges are beginning to experiment with school-wide student blogging solutions; A blog which students use for assignments the duration of their college career effectively becomes a portfolio of their work.

${ }^{7}$ From this perspective, Course Management Systems are actually subsets of Content Management Systems. Popular, general purpose, Open Source Course/Content systems include Plone, Drupal, Joomla, Sakai, Moodle, etc.

${ }^{8}$ Mark Phillipson, "Wide Open: Implementing a Class Wiki," Paper presented at Columbia University Seminar on New

Media Teaching and Learning, New York City, October 27, 2005.

${ }^{9}$ Joel Spolsky, "It's Not Just Usability," Joel on Software, September 6, 2004,

http://www.joelonsoftware.com/articles/NotJustUsability.html (Accessed June 13, 2005).

${ }^{10}$ Donald Norman, The Design of Everyday Things (New York: Basic Books, 1988).

${ }^{11}$ Lev Manovich, The Language of New Media. (Cambridge, MA: MIT Press, 2001).

${ }^{12}$ The Social Justice wiki can be found at the following URL: http://socialjustice.ccnmtl.columbia.edu.

${ }^{13}$ More information on CCNMTL can be found at the following URL: http://ccnmtl.columbia.edu/.

${ }^{14}$ Phillipp Blom, Enlightening the World: Encyclopédie, the Book That Changed the Course of History (New York: Palgrave Macmillan, 2005), 37-41.

${ }^{15}$ Blom, 48.

${ }^{16}$ Blom, 119.

${ }^{17}$ Blom, 58 -79.

${ }^{18}$ Blom, 79.

${ }^{19}$ Blom, 111.

20 The Oxford English Dictionary, 2nd ed., s.v., "Salt."

${ }^{21}$ Simon Winchester, The Meaning of Everything: The Story of the Oxford English Dictionary (New York: Oxford University Press, 2003), 43-44.

${ }^{22}$ Winchester, 54.

${ }^{23}$ Winchester, 44.

${ }^{24}$ Winchester, 107.

${ }^{25}$ Some think wikis distinguished for their anonymity-you don't need to be invited or qualified. Anyone can participate, unless the wiki is limited to members only. Actually, the $O E D$ was also open to anyone. As we now know, even certified lunatics were not barred.

${ }^{26}$ Winchester, 88-94.

${ }^{27}$ Number of active contributors - contributed at least five in a month-from Yochai Benkler, The Wealth of Networks: How Social Production Transforms Market and Freedom (New Haven: Yale University Press, 2006), 72.

${ }^{28}$ See Malcolm Gladwell, The Tipping Point (New York: Little, Brown and Company, 2000) 176-181, referring to Robin Dunbar, "Neocortex size as a constraint on group size in primates, Journal of Human Evolution (1992) vol. 20, $462-493$.

${ }^{29}$ Winchester, 56.

${ }^{30}$ Alastair Brotchie, compiler, Surrealist Games (London: Redstone Press, 1991) 141, citing Andre Breton, Second Manifesto, 1930.

${ }^{31}$ Catherine Vasseur, L'Image sans mémoire. Les cahiers du Musée national d'art moderne. 1996, printemps. 71-91.

${ }^{32}$ Christopher Alexander, A Pattern Language: Towns, Buildings, Construction (New York: Oxford University Press, 1977), 368-370.

${ }^{33}$ Roy Kozlovsky. Kozlovsky p. 2. Note: American Adventure Playgrounds are quite different.

${ }^{34}$ Kozlovsky, p. 1. 